\title{
Diacronie
}

Studi di Storia Contemporanea

$N^{\circ}$ 9, 1 | 2012

Quando la classe operaia andava in paradiso

\section{Nazionale e globale nella rinascita dell'Internazionale socialista (1945-1951)}

\section{Enrico Pugliese}

\section{(2) OpenEdition}

\section{Journals}

\section{Edizione digitale}

URL: http://journals.openedition.org/diacronie/3043

DOI: 10.4000/diacronie.3043

ISSN: 2038-0925

\section{Editore}

Association culturelle Diacronie

\section{Notizia bibliografica digitale}

Enrico Pugliese, « Nazionale e globale nella rinascita dell'Internazionale socialista (1945-1951)»,

Diacronie [Online], $\mathrm{N}^{\circ}$ 9, 1 | 2012, documento 12, Messo online il 29 janvier 2012, consultato il 20 avril 2019. URL : http://journals.openedition.org/diacronie/3043; DOI : 10.4000/diacronie.3043 


\title{
Diacronie
}

\section{2/}

\section{Nazionale e globale nella rinascita dell'Internazionale socialista (1945-1951)}

\author{
Enrico PUGLIESE*
}

Questo articolo si concentra sulle conferenze socialiste che tra il 1945 e il 1951 precedettero la rifondazione dell'Internazionale. Esso, attraverso l'archivio conservato presso l'Istituto di storia sociale di Amsterdam, affronta tre elementi di quella vicenda: la ricostruzione di una vera e propria organizzazione internazionalista, l'affermazione di nuove idee e pratiche politiche nel socialismo europeo, il sostegno dei socialisti alla governance transnazionale. Così facendo il testo evidenzia alcuni nodi cruciali per comprendere il primo decennio socialista dopo il secondo conflitto mondiale, questioni dirimenti come il rapporto con lo Stato nazione democratico e la scelta dell'integrazione europea.

\subsection{Premessa}

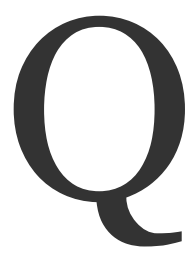

uesto lavoro si focalizza su tre fondamentali elementi della rinascita dell'Internazionale socialista nel primo decennio del secondo dopoguerra. II nucleo centrale della ricerca è costituito dalla ricostruzione strutturale del socialismo internazionalista che tenta attraverso nuove forme organizzative, fluide, liquide e in via di definizione, di rinnovare una prassi politica indebolita dalle numerose sconfitte patite nei confronti di altri influenti movimenti globali come il nazionalismo e il fascismo.

Il secondo elemento è l'affermazione di nuove vie e fratture in termini di idee e politiche che si impone ai rinati partiti socialisti per far fronte alle trasformazioni strutturali del capitale europeo e alla sovranità politica democratica emergente nel dopoguerra. 
Il terzo elemento è la formazione di un criterio tecnico di gestione della cosa pubblica, un aspetto che si evidenzia progressivamente e contraddittoriamente nel composito universo socialdemocratico soprattutto dopo la rottura del fronte di ricostruzione dell'Internazionale con l'espulsione dei partiti operai legati all'Unione sovietica e l'avvio del processo di integrazione europea.

Tenendo a mente questi riferimenti possiamo cominciare a delineare qualche tratto del primo elemento del discorso.

\subsection{L'internazionalismo socialista alla prova della ricostruzione}

Dopo la seconda guerra mondiale il fronte socialista, dilaniato dalle divisioni scaturite dai conflitti tra nazioni e dalla necessità primaria di ricostruire lo Stato, dovette fare i conti con l'affermazione di un nuovo ordine mondiale, incentrato sulla cristallizzazione dei rapporti di forza tra potenze e sull'ascesa di un nuova cornice di sovranità politica democratica ${ }^{1}$. Questo duplice imponente cambiamento ebbe qualche notevole conseguenza. In primo luogo costrinse i partiti socialisti, sotto il peso di un conflitto bipolare totalizzante, a ripensare la strategia dell'unità proletaria e antifascista. In secondo luogo impose la revisione della tradizionale opposizione alla sovranità borghese asserragliata nei confini simbolici e politici dello Stato nazione liberale. In particolare, la nuova cornice statuale, rinnovata dopo il crollo dei regimi fascisti in forme democratiche nazionali, chiamò i socialisti a un duro lavoro di riadattamento culturale e politico che fu progressivo e niente affatto indolore. È vero, infatti, che in un primo tempo in molti provarono a mantenere inalterati i tratti del vecchio antagonismo incanalandolo dentro la proposta politica di un'assemblea internazionale e internazionalista con poteri legislativi. Italiani, greci, e socialisti dell'Est, in special modo, lavorarono per instaurare uno stretto collegamento con la nascente Organizzazione delle Nazioni Unite e provarono a costruire una sorta di democrazia "reale e globale". Ma queste proposizioni, nonostante si iscrivessero nell'alveo più tradizionale e condiviso del vecchio internazionalismo, non erano destinate a suscitare un ampio apprezzamento nei grandi partiti che componevano l'Is. Buona parte dell'organizzazione, dagli inglesi ai francesi, infatti, spinta anche dalla crescente divisione in rigidi blocchi dell'Europa, pensò che appoggiare le rivendicazioni

\footnotetext{
${ }^{1}$ Sul concetto di sovranità: ZACCARIA, Giuseppe (a cura di), Lessico della politica, Roma, Edizioni Lavoro, 1987, s.v. «Sovranità» a cura di MARRAMAO, Giacomo, BOBBIO, Norberto, MATTEUCCI, Nicola, PASQUINO, Gianfranco (a cura di), Dizionario di politica, Milano, TEA, s.v. «Sovranità» a cura di MATTEUCCI, Nicola, 1990, pp. 1079-1088; BASSO, Lelio, Il principe senza scettro, Milano, Feltrinelli, 1958.
} 
più tradizionali di italiani ed est europei significasse riprodurre i limiti di una vecchia prassi: il velleitarismo nella ricerca di soluzioni concrete e alternative al quadro statuale in vigore e l'incapacità di garantire una reale unità di vedute. L'evidenza di queste storiche fragilità sconsigliò, quindi, i partiti inglese, francese e tedesco dal riproporre vecchi modelli già sconfitti dalle guerre e suggerì ad essi un deciso meccanismo di rinnovamento del pensiero e della prassi politica socialista. L'opzione, sintetizzata da Guy Mollet, leader dei socialisti francesi, in «seul un internationalisme constructif [...] permettra la renaissance d'une classe ouvrière majeure, unie dans une nouvelle Internationale et apte à sa mission historique» fu quindi perseguita fino al cambiamento degli orizzonti politici del socialismo². Questa lucida consapevolezza, cioè, condivisa dai più importanti protagonisti del socialismo europeo, sia riguardo le forme che l'internazionalismo avrebbe dovuto assumere sia riguardo le tendenze che esso avrebbe dovuto assecondare, portò al passaggio da una politica pensata ed espressa principalmente sul piano locale o globale a una spiccata predilezione per la politica nazionale o europea. In questo senso, lo Stato nazione, rinato dalle ceneri del conflitto, fu issato a pilastro della nuova edificazione ideologica e valoriale del socialismo europeo divenendo il principale asse della politica socialista.

La "costruzione" di questo nuovo internazionalismo basato sulla forza dello Stato nazione cominciò dall'Assemblea di Londra nel marzo del 1945 (a guerra ancora in corso). Nella capitale inglese si riunirono 13 partiti di ispirazione socialista, quelli che avrebbero costituito l'embrione originario della nuova Is. Lì grazie all'attivismo e all'energia profusi dal British Labour Party (Blp) vennero poste le basi delle relazioni interpartitiche che avrebbero segnato gli anni seguenti. Il partito laburista, forte di un ampio consenso popolare, dettò l'agenda politica e soprattutto le nuove linee di frattura ideologica all'interno della comunità internazionale. In primo luogo, gli inglesi rifiutarono l'integrazione dell'economia europea secondo costruzioni di entità statuali unitarie e la formazione di un organismo sovranazionale che assorbisse gli spazi di sovranità degli stati nazione. In secondo luogo negarono la costituzione di un superpartito o sovrapartito socialista come cinghia di trasmissione con le nuove realtà sovranazionali. Essi cioè impostarono la nascente Is come un luogo di discussione e scambio di considerazioni, un centro di connessione di legittimi interessi per favorire la regionalizzazione delle relazioni economiche e politiche in Europa e nel mondo. Assieme alle tradizionali tendenze del vecchio internazionalismo, cioè, essi si produssero in una riedificazione del socialismo e della sua prassi su basi innovative. In questo senso, le suggestioni di una riforma in senso democratico/assembleare

2 MOLLET, Guy, Témoignages 1905-1975, Paris, Fondation Guy Mollet, 1977, p. 60. 
lasciarono presto spazio all'apertura di una stagione nuova di composizione degli interessi di classe nazionali ed europei, all'idea di una riforma statuale che sostituisse l'idea precedente del suo completo abbattimento3.

Questi tasselli che si affermarono sempre con più forza nelle conferenze che tra il 1945 e il 1947 si succedettero per iniziativa inglese scandirono i modi e i tempi della nuova organizzazione consegnando il variegato mondo socialista alla leadership laburista. Questa supremazia inglese si espresse principalmente nella delimitazione di un moderno spazio politico quale primo elemento di una nuova identità e di un nuovo senso politico progressista. In questo quadro va letta la decisione dei laburisti di escludere il partito socialdemocratico tedesco (Spd) dalle riunioni per la fondazione della nascente architettura socialista internazionale (la Spd verrà inclusa nell'Is solo in un secondo tempo) ${ }^{4}$. In quel provvedimento infatti si affermava un paradigma politico cruciale per il futuro dell'organizzazione: l'idea di riconoscere come avversari politici i partiti appartenenti alle nazioni nemiche o concorrenti del Regno Unito (la condanna del partito tedesco scaturì direttamente dalle sessioni della direzione politica del Labour)5. Vennero proposti come criteri di inclusione/esclusione dalla comunità socialista due elementi: la coerenza delle scelte politiche globali operate dai partiti socialisti e la loro appartenenza nazionale. Si affermò così un clivage patriottico più che classista che conteneva un alto potenziale di disgregazione poiché ruotava attorno al rispetto dei principi dell'appartenenza nazionale dando meno spazio alla costruzione di alternative sovranazionali per favorire l'abbattimento delle frontiere economico politiche ${ }^{6}$. Il percorso costituente del socialismo partì quindi dalla razionalizzazione del numero e della qualità dei partecipanti provando a rendere l'organizzazione più omogenea e concorrenziale, in termini di coerenza, con lo spazio politico comunista. Questa scelta di dividere e restringere il fronte proletario comportò, tuttavia, una resa

\footnotetext{
3 Sul periodo egemonico inglese vedi IISH, Amsterdam, International Socialist Archives, 234235, London, Clacton on Sea, Bornemouth, verbali delle conferenze; sulla conferenza di Londra del 3-4-5 Marzo 1945 vedi anche DEVIN Guillaume, L'Internationale socialiste (1945-1976): Politique et Ethique du Socialisme International, thèse pour le Doctorat en science politique (dir. H. Portelli), Université de Paris X-Nanterre, Paris, Presses de la Fondation nationale des sciences politiques, 1988.

4 La decisione di includere la Spd andò di pari passo con la ri-legittimazione della Repubblica Federale Tedesca nel quadro dell'alleanza antisovietica. Una decisa accelerazione in tal senso venne dalla Conferenza di Zurigo nel 1947. Su questo vedi IISH, Amsterdam, Socialist International Archives, 236, Conference of Zurich, corrispondenza Schumacher-Healey, Hannover 7 aprile - Londra 27 aprile 1947.

5 Vedi GLEES, Anthony, Exile Politics during the Second World War: The German Social Democrats in Britain, New York, Oxford University Press, 1982.

${ }^{6}$ MISGELD, Klaus, Die Internationale Gruppe demokratischer Sozialisttichen in Stockholm 1942- 1945, Almquist and Wiksell International, Uppsala 1976, p. 111, cit. in DEVIN, Guillaume, op. cit., pp. 2-29.
} 
dei conti interna con quanti non volevano rotture traumatiche né delimitazioni forzate. Ben presto quindi si palesò una linea alternativa incardinata nelle proposte del polo socialdemocratico scandinavo. Il "gruppo di Stoccolma" composto da svedesi, norvegesi e tedeschi, diede forma ad una progetto incentrato sulla formazione di una comunità integrata e comprensiva di tutte le realtà del socialismo, senza alcuna esclusione preventiva, al fine di ottenere la piena «unità del movimento operaio». In base a questi propositi, i "socialdemocratici" si dissero pronti a definire un programma di riforme economiche che fosse d'ispirazione per l'intera comunità socialista internazionale7. La veloce progressione del nuovo ordine che prospettava la divisione del mondo in più sfere d'influenza suggeriva loro delle aperture sia sull'adesione dei socialdemocratici tedeschi all'Is che sulla costruzione di forme progressive di coordinamento europeo ${ }^{8}$. Il progetto di ricostruzione scandinavo, cioè, ricavava uno spazio per un'idea di Europa come nuova frontiera comune delle politiche sociali e inclusione dei paesi in conflitto nel progetto condiviso della pace e delle riduzione delle disuguaglianze.

Questa lettura scandinava delle vicende aveva un forte fondamento in una prospettiva di incanalamento e depotenziamento del conflitto di classe. Anche l'idea di dialogare con i comunisti creando materialmente un'Europa dei socialisti rispondeva al tentativo di formare una istituzione statuale autodeterminata e autosufficiente, una terza forza come espressione pacifista tout court. Ma questo tipo di pensiero poteva incontrare il favore di tutta l'Is? E soprattutto poteva bastare agli inglesi? La risposta è senz'altro no. Sebbene, infatti, vi fossero alcune condizioni utili a creare una condivisione maggiore sul progetto scandinavo, alcuni fondamentali tasselli di quel paradigma, in primo luogo il terzaforzismo, ne impedivano la riuscita. Gli inglesi, nonostante giudicassero affascinante una certa propensione alla riorganizzazione del conflitto da parte dei socialdemocratici scandinavi, non erano certamente propensi a costruire realtà sovranazionali indipendenti dal potere USA, né altra forme di organizzazioni sovrane, democratiche e autosufficienti dal blocco occidentale. Per questo essi impedivano ogni tipo di accelerazione che potesse prefigurare la nascita di

\footnotetext{
7 Sulla storia della socialdemocrazia svedese vedi: KORPI, Walter, Il compromesso svedese 1932-1976. Classe operaia sindacato e Stato nel capitalismo del Welfare, Bari, De Donato, 1982. Sul modello sociale scandinavo vedi anche il più recente BORIONI, Paolo (a cura di), Il Welfare Scandinavo, Roma, Carocci, 2004.

8 Su questo punto vedi DEVIN, Guillaume, op. cit., pp. 2-29. A partire dalla fusione dei socialdemocratici e dei comunisti tedeschi nella zona sovietica (che segnò l'irrigidimento del controllo politico comunista) e con la formazione di una zona angloamericana sul suolo germanico gli stessi inglesi cominciarono a lavorare per rompere l'isolamento tedesco. Ma, nonostante quel cambiamento di rotta, i fondamenti patriottici legati alle economie nazionalizzate della guerra e connessi alla cultura politica socialdemocratica ed in particolare alle politiche sociali del welfare state rimasero presenti nel nucleo fondativo dell'Internazionale socialista (forse con l'unica eccezione dei socialisti francesi).
} 
una soggettività politica socialista davvero coesa e transnazionale. Essi rifiutavano ad esempio l'idea che i socialisti potessero formulare unitariamente anche poche e selezionate "linee guida" in materia economica, convinti che ogni stato - e ogni partito - dovesse proporre e seguire la propria ricetta di modernizzazione e di sviluppo limitandosi a intrattenere relazioni fraterne e proficue con gli altri soggetti della politica internazionale. In questo senso, l'Is doveva svolgere una semplice funzione di mediazione e incontro tra le differenti proposte economiche dei partiti nazionali, non uniformandole ma proponendole alla platea socialista come modello di gestione possibile. Ciò evidentemente scavava un solco tra il modello inglese di Internazionale e quello scandinavo rischiando di compromettere il cammino unificante dell'Is, tanto più che dietro a queste differenze di impostazione e di ideologia si celavano visioni divergenti sulla composizione stessa del movimento 9 .

Di fronte a questo quadro che vedeva gli inglesi come ostacolo a un tipo di sviluppo partitico del movimento e a un'idea forte di Internazionale, era quindi necessario trovare nuove energie unificanti, secondo una proposta politica condensata, ancora una volta, nella constatazione di Guy Mollet:

Le parti socialiste est à la fois national et International. Parce qu'il n'y a pas de travail libre dans une nation asservie ou sujette; parce que les travailleurs, que les abus du capitalisme tendaient à rejeter hors de la patrie, s'y sont intégrés euxmêmes par une siècle et demi d'efforts et des sacrifices; que la patrie est aujourd'hui leur bien et [pour] une grande partie leur œuvre, et qu'ils sont déterminés à la défendre. ${ }^{10}$

La dialettica ristretta tra gli inglesi e gli scandinavi, sembrava sottintendere il socialista francese, rischiava di non far emergere il vero nodo della questione: il cambiamento delle prospettive richiesto dalle masse nazionalizzate al socialismo internazionalista. I lavoratori, secondo Mollet, avevano scelto l'appartenenza nazionale come rifugio di fronte agli "abusi" del capitalismo e i socialisti avevano il compito di confrontarsi con questa realtà, di comprenderne le ragioni e di tenerne conto nell'elaborazione della propria politica internazionalista ${ }^{11}$. Ma per compiere questo passaggio c'era bisogno di un apporto più ampio dei partiti appartenenti all'Is, di una dose di riflessioni più accurate e condivise sull'identità e sulla politica del nuovo

9 IISH, Amsterdam, International Socialist Archives, 234, Socialist Conference Clacton on Sea, May 15th-2oth 1946, Corrispondenza Morgan Philips, 21 February 1946.

${ }^{10}$ MOLLET, Guy, op. cit., p. 62.

${ }^{11}$ DEVIN, Guillaume, op. cit., pp. 2-29. 
socialismo. Per questa ragione sarebbe stato utile che i grandi partiti socialisti europei avessero elaborato una propria politica sia sull'Internazionale che sull'Europa. Che la SFIO (Section française de l'Internationale ouvrière) si fosse messa alla guida di un convinto fronte europeista e che il Psi (Partito socialista italiano) avesse portato dentro l'Is i suoi contenuti antagonisti ${ }^{12}$. Da questa dialettica, radicale e costruttiva, sarebbe potuto venire un impulso alla chiarificazione del percorso della nascente Is.

\subsection{Convergenze e divisioni}

Quando la SFIO e il Psi entrarono in scena la nascente Internazionale si componeva fondamentalmente di tre fronti più o meno coesi. Il primo, alla guida dell'organizzazione, era formato dai laburisti inglesi che intrattenevano rapporti diplomatici e di vicinanza con gli altri gruppi. Il secondo, il più coeso, era invece costituito dai socialisti dell'Europa orientale e dal Partito socialista italiano, soggetti politici propensi alla collaborazione sistematica con i partiti comunisti europei. Infine il terzo, una sorta di opposizione interna al sistema di governo inglese, era formato da una galassia di partiti di grande tradizione che tuttavia non riuscivano a trovare una linea comune. Di quest'ultimo filone, attraversato da divisioni interne che ne impedivano una reale ascesa alla guida del movimento, facevano parte i socialisti francesi (la SFIO), che mantenevano forti legami con i socialisti belgi e olandesi, i partiti scandinavi, e il partito tedesco, sia pure posto in posizione subordinata e costretto a rincorrere l'approvazione dell'Internazionale (nel 1947). Ma questa composizione degli equilibri era a dir poco traballante. Il montare della guerra fredda e l'affermazione di un paradigma e di una prassi politica che parlava di riedificazione nazionale in senso democratico e liberale e di Stato nazione "commercializzato" quale pilastro di un nuovo ordine mondiale, chiamavano all'esclusione dei partiti socialisti vicini all'Unione sovietica ${ }^{13}$. Questo passaggio decisivo e periodizzante per il nostro racconto avvenne con relativa tempestività. Infatti, in corrispondenza della nascita del COMINFORM, l'organizzazione di collegamento tra i diversi partiti comunisti europei guidata dall'URSS, fu ratificata l'uscita di quei partiti che intrattenevano rapporti di vicinanza con l'altra faccia del proletariato. Tra il 1947 e il 1949, un biennio che segnò il

${ }_{12}$ MATTERA Paolo, Il partito inquieto. Organizzazione, passioni e politica dei socialisti italiani dalla Resistenza al miracolo economico, Roma, Carocci, 2004. Sui rapporti tra inglesi ed italiani vedi NENNI, Pietro, Tempo di guerra fredda. Diari 1943-1956, Milano, SugarCo, 1981. ${ }^{13}$ MILWARD, Alan S., The Reconstruction of Western Europe, 1945-51, Berkeley, University of California Press, 1984; ID., The European Rescue of the Nation State, London, Routledge, 2000; ROSECRANCE, Richard, The Rise of the Trading State: Commerce and Conquest in the Modern World, New York, Basic Books, 1986. 
rafforzamento di un'identità socialdemocratica dell'assemblea internazionale (nel frattempo divenuta COMISCO, Committee of the International Socialist Conference, in seguito alla conferenza di Zurigo del 1947) furono espulsi i partiti socialisti dell'Est e soprattutto il Partito socialista italiano. Questa scelta assumeva particolare importanza per due ragioni. La prima, di ordine più strettamente politico, era che l'internazionale decise di non tenere conto dell'evoluzione che si stava profilando proprio in quel 1948 nel Psi. L'Is mostrò cioè di non credere nella capacità della "direzione centrista" di Alberto Jacometti e di Riccardo Lombardi, nata dopo la débâcle elettorale del 14 aprile, di affermare una linea politica di "autonomia", improntata a una maggiore distanza dal Pci (ma senza chiusure preconcette) e a un nuovo avvicinamento ai partiti socialisti europei, in particolar modo al Labour. La seconda ragione, di ordine più strategico, era invece legata al carattere simbolico dell'espulsione del Psi. Con l'allontanamento del partito italiano, considerato dall'Internazionale come una frontiera aperta tra il mondo comunista e il mondo socialista, un crocevia intenso di relazioni tra blocchi, si pose un pesante sbarramento a ogni prospettiva di dialogo ${ }^{14}$. Ancora una volta cioè nella storia del socialismo europeo il criterio della differenza prevalse su quello della condivisione e dell'unità. In altre parole, si riconobbe prima il nemico dell'amico (il binomio schmittiano), affermando per questa via la nuova identità collettiva. Il socialismo fu allora riconvertito a un'idea di autonomia dal comunismo come unica via politica possibile al governo delle nazioni e su questa primaria prassi di azione si basarono tutte le idee chiave su cui esso si andò a riedificare l'Internazionale socialista. Questa accelerazione prese vigore tra la conferenza di Zurigo del 1947 e quella di Copenaghen del 1950. In quella breve fase, si definirono i modi e i tempi della ricostruzione fattiva e concreta della struttura politica della nuova Is, organigramma, dirigenti, gruppi e sottogruppi, e si puntò alla modernizzazione della proposta politica socialista ${ }^{15}$.

Il risultato più importante di questa azione fu sancito dal passaggio da un orizzonte politico incentrato sul primato della democrazia economica (elaborazione delle riforme e delle strategie per il suo raggiungimento) a una prospettiva valoriale che parlava della costruzione di una democrazia politica. Là dove democrazia politica stava per l'irrinunciabile godimento e la supremazia dei diritti politici (civili, si direbbe oggi) su

${ }_{14} \mathrm{Su}$ questa fase vedi: MATTERA, Paolo, op. cit., pp. 151-162; SCIROCCO, Giovanni, Politique d'abord: il PSI, la guerra fredda e la politica internazionale (1948-1957), Milano, Unicopli, 2010, pp. 38-71.

15 IISH Amsterdam, Socialist International archives, 235, International socialist conference Zurich June 1947, minutes; IISH, Amsterdam, International Socialist Archives, 237, COMISCO, Anversa 28 novembre - 2 dicembre 1948; vedi anche IISH, Amsterdam, International Socialist Archives, 265, Comisco Meetings. 1948-50; IISH, Amsterdam Amsterdam, Communism. 194853,1956-61, COMISCO Paris 10th-11th December 1949, Resolution; IISH, International Socialist Archives, Amsterdam, 240, Conference of Copenaghen 1950, verbali. 
quelli economici, per la preminenza dell'individuo, della sua sfera di libertà, sui diritti collettivi, e sul principio dell'uguaglianza economica ${ }^{16}$. Questo paradigma fu inciso a caratteri cubitali nel Manifesto di Francoforte del 1951, la carta nella quale vennero rese pubbliche le priorità e i valori del nuovo socialismo europeo (e mondiale):

\section{Political democracy}

2. Without freedom there can be no Socialism. Socialism can be achieved only through democracy. Democracy can be fully realised only through Socialism.

Democracy is government of the people, by the people, for the people. It must secure:

- the right of every human being to a private life, protected from arbitrary invasion by the state.

- Political liberties like freedom of thought, expression, education, organisation, and religion.

- The representation of the people through free elections, under universal, equal and secret franchise.

- Government by the majority and respect for the rights of the minority.

- The equality before the law of all citizens, whatever their birth, sex, language creed and colour.

- Right to cultural autonomy for groups with their own language.

- An independent judiciary system; every man must have the right to a public trial before an impartial tribunal by due process of law..$^{17}$

È evidente che non c'è il necessario spazio per sviscerare tutte le conseguenze di questo passaggio epocale che ebbe il carattere di una vera e propria rivoluzione delle prospettive per il socialismo europeo. Ci basti, allora, evidenziare due elementi delle trasformazioni inedite che suscitò la democrazia politica nell'universo politico socialista. In primo luogo essa segnò la fine dell'antagonismo rituale del socialismo con lo stato nazione liberale e l'edificazione di un nuovo paradigma che, nel regime democratico del dopoguerra, divenne piena condivisione della sovranità politica sancita dalle costituzioni nate dalla resistenza. E dunque: centralità del parlamento, equilibrio dei poteri e sovranità del popolo e per il popolo esercitata mediante rappresentanza. In

${ }^{16}$ IISH, Amsterdam, Conference of Frankfurt, 241, Declaration of the principles of democratic socialism.

${ }_{17}$ IISH, Amsterdam, International Socialist Archives, 241, Conference of Frankfurt, Declaration of the principles of democratic socialism, Political Democracy. 
secondo luogo, essa comportò la destrutturazione e la ricostruzione del pensiero e della prassi d'azione socialista riguardo alla relazione tra nazionale e globale. Con la democrazia politica, infatti, si affermò un nuovo paradigma del mondo, dei suoi poteri e dei suoi rapporti che vide lo Stato nazionale come primo garante della libertà individuale, le istituzioni internazionali (l'ONU, il Fondo monetario) come organi funzionali allo sviluppo di una democrazia pluralistica globale chiamata a controllare l'attuazione dei precetti liberali. La nuova democrazia transnazionale, cioè, doveva essere basata su uno stabile ancoraggio allo Stato nazione (fornitore di servizi, di identità e di cittadinanza) e su contropoteri definiti e riconoscibili. L'inversione di rotta rispetto al precedente paradigma socialista che tendeva ad escludere, o quantomeno a ridimensionare, la politica nazionale per privilegiare la prassi di azione locale e/o globale e a combinare nella prassi organizzativa gerarchia e egualitarismo era davvero notevole. Emblematico di questo nuovo modo di pensare era, ad esempio, l'obiettivo condiviso della costruzione europea, fissato tra le priorità del socialismo democratico. Questo progetto, nutrito dalla potente spinta al raggiungimento di una pace stabile in Europa, era, infatti, visto come un tentativo di mediazione tra la propensione storica dei socialisti all'abbattimento delle barriere nazionali e un paradigma liberale e liberista contrario alla democrazia economica di impronta comunista. L'integrazione europea, cioè, prefigurava una riduzione programmatica del conflitto tra il capitale e il lavoro, un'idea tipicamente socialdemocratica sempre più in voga nell'Internazionale socialista. Era evidente con questo che il socialismo si preparava a riemergere dalle ceneri delle guerre attraverso una politica di conciliazione che si esplicitava in due modi. Da una parte per via dell'elevazione della libertà individuale a principio guida della comunità internazionalista e a diritto inviolabile del cittadino, dall'altra per via dell'unificazione europea quale orizzonte privilegiato della costruzione sovranazionale socialista.

\subsection{Tecnocrazia e governance europea}

Il terzo e ultimo punto della nostra disamina tocca alcune delle implicazioni più importanti dell'elevazione della formazione europea a valore unificante del socialismo. Essa infatti, per quanto importante, non costituiva la sola variazione di una prospettiva storico/politica ma voleva dire affermare una nuova prassi di governo dell'Europa, la possibilità di ridurre la democrazia sovranazionale ad amministrazione dei processi capitalistici secondo un modello di "governance apartitica o sovrapartitica" tecnico capitalistica. 
Nel contesto mutato dello Stato nazione commercializzato (o Trading State), infatti, era convinzione delle forze politiche che guidavano il processo di europeizzazione che fosse necessario garantire un livello di scambi adeguato, salvaguardare, con l'abbattimento delle frontiere, il carattere capitalistico dei sistemi produttivi nazionali, gli affari tra potenze occidentali. I soggetti conservatori in auge nel fronte favorevole all'europeizzazione pensavano, cioè, che senza l'apertura al mercato, senza la libera circolazione delle merci, e della manodopera, senza legare il progetto d'integrazione economica a un'idea liberista dello sviluppo non sarebbe stato possibile ricostruire gli Stati nazione e impedire quindi rivalse e nuove guerre. Essi quindi puntavano a ratificare quel processo economico e con esso ad affermare lo status liberista dello Stato nazione. In quest'ottica, la gestione tecnicistica e prettamente funzionalistica del governo economico europeo assicurava, oltre e al di là della democrazia costituzionale del dopoguerra sostenuta dai socialisti, la stabilità del processo di formazione europea, la sopravvivenza dello Stato nazione e il persistere del sistema capitalistico. In questo senso, il prospettato cambiamento della sovranità in sede europea non prefigurava una semplice dialettica tra idee "progressiste o conservatrici” dell'integrazione ma un conflitto radicale tra due modelli differenti di sovranità, una contraddizione fondamentale che le forze antagoniste, comprese quelle socialiste, potevano amplificare o depotenziare. La possibile declinazione "liberista e reazionaria" del progetto europeo poneva, cioè, il socialismo europeo di fronte a un bivio: o ratificare il proprio ingresso in una prospettiva di riforme di stampo liberista o apportare con urgenza un proprio contributo alla nascente unità, compiendo un intervento politico in grado di distogliere l'attenzione dall'Europa dei mercati per connotare in senso progressista il progetto di integrazione. Mentre la prima ipotesi voleva dire iniziare un confronto interno per aderire a un sistema economico lontano dalla tradizione socialista, la seconda via, la via socialista all'Europa, significava imporre il ribaltamento del tavolo delle trattative, elevare la politica a guida del processo di edificazione europea ${ }^{18}$. Mentre l'Europa già pensata dai conservatori non chiedeva altro ai socialisti che prendere parte ai meccanismi in atto nel capitalismo nazionale e europeo, l'Europa socialista era tutta da immaginare ed esigeva una straordinaria capacità di esercitare la propria egemonia culturale sui soggetti economici e politici nazionali ed europei.

Non possiamo evidentemente qui riportare tutto il dibattito che portò a sciogliere il nodo europeista. Possiamo però certamente valutare come i socialisti si predisposero

$18 \mathrm{Su}$ questo punto vedi PHILIP, André, L'Europe unie et sa place dans l'économie internationale, Paris, PUF, 1953. 
alla soluzione del problema. Per dirimere la questione, infatti, fu creato un gruppo di studio sull'Europa, composto da personalità politiche inviate dai partiti come lo svedese Kaj Åke Björk, il francese Simon Grumbach, l’italiano Mario Zagari, il belga Victor Larock, il tedesco Erik Nolting. Questo gruppo, nato per impostare una posizione politica socialista sull'Europa, valutò i processi di integrazione in atto e discusse delle fragilità che essi mostravano. Ciò che suscitò, in questo senso, il più ampio dibattito fu l'assenza di democrazia rappresentativa nel piano Schuman, un progetto fortemente caldeggiato dal diplomatico e funzionario francese Jean Monnet e imperniato sull'idea di costruire un'Europa dei mercati attraverso un meccanismo di governance ademocratica $^{19}$. Le reazioni a questo genere di proposta furono di iniziale scalpore e contrarietà, sentimenti che, tuttavia, furono presto abbandonati di fronte alle crescenti difficoltà dell'integrazione economica. Si fecero allora largo delle timide aperture che in un'ottica riformista e dialogante portarono alla proposta di democratizzare, almeno in parte, il piano Schuman ${ }^{20}$. In questo senso, l'idea dei socialisti fu quella di associare al progetto di integrazione economica di Schuman e Monnet, l'assemblea parlamentare di nomina politica del Consiglio d'Europa, una istituzione nata il 5 maggio 1949 con lo scopo di favorire la creazione di uno spazio democratico e giuridico comune. La nuova assemblea, appoggiata da tutti $\mathrm{i}$ socialisti del gruppo, rivelava un approccio di mediazione tra il tecnicismo delle istituzioni economiche e il piano politico delle vicende e prefigurava l'idea di creare una classe dirigente specializzata sul tema dell'unificazione europea, una sorta di personale né prettamente politico, né prettamente tecnico economico, in grado di sviluppare consenso nelle alte sfere diplomatiche, di dialogare con l'alta dirigenza dei capitalismi nazionali, e allo stesso tempo di parlare ad un ceto ristretto di addetti che si andava formando intorno alla CECA (Comunità europea del carbone e dell'acciaio). I partiti, secondo questa prospettiva, dovevano cioè prepararsi all'inevitabile europeizzazione del capitale, interagire, attraverso i piani economici, con esso e contemporaneamente riuscire a spiegare, sul piano nazionale, la perdita di potere decisionale delle rappresentanze tradizionali $^{21}$. Si trattava di un compito davvero arduo da affrontare poiché c'era bisogno di operare una sintesi complessa tra punti di vista politici, contrari alla perdita

19 Sullidea di una governance ademocratica e sulle ragioni dell'opposizione socialista vedi OURS, Paris, Archive Guy Mollet, 110, Mouvement socialiste pour les États-Unis d'Europe, Strasbourg 17-18-19 Novembre 1950, Le problème de la création d'une réelle autorité politique européenne supranationale.

${ }^{20}$ IISH, Amsterdam, Socialist International Archives, 583, European Unity 1951-1953, GROUPE D'ÉTUDE SUR L’UNITÉ EUROPÉENNE, Rapport presenté au nom du Group au Conseil de l'Internationale à Bruxelles, le 14 décembre 1951, par M. Van der Goes van Naters, rapporteur. ${ }^{21}$ Ibidem. 
della sovranità nazionale, ed esigenze di natura tecnica che chiedevano l'allineamento degli Stati nazione nel quadro liberista che si andava edificando. Si poneva una contraddizione di fondo che, come si può evincere in modo molto chiaro dai dibattiti del gruppo di studio sull'Europa, non poteva essere risolta senza mettere a rischio i piani di integrazione. Non stupisce, quindi, che di fronte a tale intricato nodo, $\mathrm{i}$ socialisti non furono capaci di risolvere la questione finendo per farsi trascinare dentro un progetto non del tutto condiviso. Ciò ebbe pesanti conseguenze sia per l'Europa, che mancò del fondamentale supporto delle istanze socialiste, che per quella cultura politica che ancora per molti anni fu prigioniera del conflitto tra l'ordine tecnico dei piani e la necessità di integrare le masse nazionalizzate nel corpo istituzionale europeo. Neanche negli anni di espansione dei diritti e della democrazia, negli anni d'oro del capitalismo europeo, il socialismo, fautore della democrazia politica, fu in grado di risolvere la contraddizione e di riformare l'Europa, e le forme di governance tecnico capitalistica ad essa connesse. Questo problema di fondo sarebbe quindi giunto immutato fino all'attuale crisi economica mondiale trascinando con sé molte delle difficoltà e delle fragilità evidenziate cinquanta anni prima nei dibattiti dell'Internazionale socialista. 


\section{* L'autore}

Enrico Pugliese si è laureato nel 2008 in storia contemporanea presso l'università di Roma Tre con una tesi su Il Movimento di Unità Popolare 1953-1957. È attualmente dottorando presso l'Università di Reading. Collabora con la Fondazione Lelio e Lisli Basso di Roma. È stato PhD visiting presso l'Università di Amsterdam con una ricerca su Il nazionale e l'internazionale nel pensiero socialista europeo del secondo dopoguerra.

URL: < http://studistorici.com/progett/autori/\#Pugliese >

\section{Per citare questo articolo:}

PUGLIESE, Enrico, «Nazionale e globale nella rinascita dell'Internazionale socialista (1945-1951)», Diacronie. Studi di Storia Contemporanea: Quando la classe operaia andava in paradiso, 13/2/2012,

URL:<http://www.studistorici.com/2012/02/13/pugliese_numero_9/ >

Diacronie Studi di Storia Contemporanea of www.diacronie.it

Risorsa digitale indipendente a carattere storiografico. Uscita trimestrale. redazione.diacronie@hotmail.it

Comitato di redazione: Marco Abram - Giampaolo Amodei - Jacopo Bassi - Luca Bufarale - Alessandro Cattunar - Alice De Rensis Barbara Galimberti - Deborah Paci - Fausto Pietrancosta - Martina Sanna - Matteo Tomasoni - Luca Zuccolo

Diritti: gli articoli di Diacronie. Studi di Storia Contemporanea sono pubblicati sotto licenza Creative Commons 2.5. Possono essere riprodotti a patto di non modificarne i contenuti e di non usarli per fini commerciali. La citazione di estratti è comunque sempre autorizzata, nei limiti previsti dalla legge. 